\title{
Dissipative Sturm-Liouville Operators with Transmission Conditions
}

\author{
Hüseyin Tuna ${ }^{1}$ and Aytekin Eryılmaz ${ }^{2}$ \\ ${ }^{1}$ Department of Mathematics, Mehmet Akif Ersoy University, 15100 Burdur, Turkey \\ ${ }^{2}$ Department of Mathematics, Nevsehir University, 50300 Nevsehir, Turkey
}

Correspondence should be addressed to Aytekin Eryılmaz; eryilmazaytekin@gmail.com

Received 7 December 2012; Accepted 11 February 2013

Academic Editor: Lucas Jódar

Copyright ( 2013 H. Tuna and A. Eryllmaz. This is an open access article distributed under the Creative Commons Attribution License, which permits unrestricted use, distribution, and reproduction in any medium, provided the original work is properly cited.

In this paper we study dissipative Sturm-Liouville operators with transmission conditions. By using Pavlov's method (Pavlov 1947, Pavlov 1981, Pavlov 1975, and Pavlov 1977), we proved a theorem on completeness of the system of eigenvectors and associated vectors of the dissipative Sturm-Liouville operators with transmission conditions.

\section{Introduction}

Spectral theory is one of the main branches of modern functional analysis and it has many applications in mathematics and applied sciences. There has recently been great interest in spectral analysis of Sturm-Liouville boundary value problems with eigenparameter-dependent boundary conditions (see [1-14]). Furthermore, many researchers have studied some boundary value problems that may have discontinuities in the solution or its derivative at an interior point $c$ [15-19]. Such conditions which include left and right limits of solutions and their derivatives at $c$ are often called "transmission conditions" or "interface conditions." These problems often arise in varied assortment of physical transfer problems [20].

The spectral analysis of non-self-adjoint (dissipative) operators is based on ideas of the functional model and dilation theory rather than the method of contour integration of resolvent which is studied by Naimark [21], but this method is not effective in studying the spectral analysis of boundary value problem. The functional model technique acts a part on the fundamental theorem of Nagy-Foiaş. In 1960s independently from Nagy-Foiaş [22], Lax and Phillips [23] developed abstract scattering programme that is very important in scattering theory. Pavlov's functional model [24-28] has been extended to dissipative operators which are finite dimensional extensions of a symmetric operator, and the corresponding dissipative and Lax-Phillips scattering matrix was investigated in some detail [5-14, 22-27, 29, 30]. This theory is based on the notion of incoming and outgoing subspaces to obtain information about analytical properties of scattering matrix by utilizing properties of original unitary group. By combining the results of Nagy-Foiaş and LaxPhillips, characteristic function is expressed with scattering matrix and the dilation of dissipative operator is set up. By means of different spectral representation of dilation, given operator can be written very simply and functional models are obtained. The eigenvalues, eigenvectors and spectral projection of model operator are expressed obviously by characteristic function. The problem of completeness of the system of eigenvectors is solved by writing characteristic function as factorization.

The purpose of this paper is to study non-self-adjoint Sturm-Liouville operators with transmission conditions. To do this, we constructed a functional model of dissipative operator by means of the incoming and outgoing spectral representations and defined its characteristic function, because this makes it possible to determine the scattering matrix of dilation according to the Lax and Phillips scheme [23]. Finally, we proved a theorem on completeness of the system of eigenvectors and associated vectors of dissipative operators which is based on the method of Pavlov. While proving our results, we use the machinery of $[5,7-10]$. 


\section{Self-Adjoint Dilation of Dissipative Sturm-Liouville Operator}

Consider the differential expression

$$
l(y)=-y^{\prime \prime}+q(x) y, \quad x \in[a, c) \cup(c, b),
$$

where $I_{1}:=[a, c), I_{2}:=(c, b)$ and $I=I_{1} \cup I_{2}, q(x)$ is realvalued function on $I$, and $q \in L_{\mathrm{loc}}^{1}(I)$. The points $a$ and $c$ are regular and $b$ is singular for the differential expression $l(y)$. Moreover $q(c \pm):=\lim _{x \rightarrow c \pm} q(x)$ one-sided limits exist and are finite.

To pass from the differential expression $l(y)$ to operators, we introduce the Hilbert space $H=L^{2}\left(I_{1}\right) \oplus L^{2}\left(I_{2}\right)$ with the inner product

$$
\langle f, g\rangle_{H}:=\gamma_{1} \gamma_{2} \int_{a}^{c} f_{1} \overline{g_{1}} d x+\delta_{1} \delta_{2} \int_{c}^{b} f_{2} \overline{g_{2}} d x,
$$

where

$$
\begin{aligned}
& f(x)=\left\{\begin{array}{ll}
f_{1}(x), & x \in I_{1} \\
f_{2}(x), & x \in I_{2}
\end{array} \quad \in H,\right. \\
& g(x)=\left\{\begin{array}{ll}
g_{1}(x), & x \in I_{1} \\
g_{2}(x), & x \in I_{2}
\end{array} \in H,\right.
\end{aligned}
$$

and $\gamma_{1}, \gamma_{2}, \delta_{1}$, and $\delta_{2}$ are some real numbers with $\gamma_{1} \gamma_{2}>0$ and $\delta_{1} \delta_{2}>0$.

Let $L_{0}$ denote the closure of the minimal operator generated by (1) and by $D_{0}$ its domain. Besides, we denote the set of all functions $f(x)$ from $H$ such that $f, f^{\prime} \in$ $A C_{\text {loc }}(I), f(c \pm), f^{\prime}(c \pm)$ one-sided limits exist and are finite and $l(y) \in H ; D$ is the domain of the maximal operator $L$. Furthermore, $L=L_{0}^{*}$ [21].

For two arbitrary functions $y(x), z(x) \in D$, we have Green's formula

$$
\begin{aligned}
\int_{a}^{b} l(y) \bar{z} d x-\int_{a}^{b} y \overline{l(z)} d x= & \gamma_{1} \gamma_{2}[y, z]_{c^{-}}-\gamma_{1} \gamma_{2}[y, z]_{a} \\
& +\delta_{1} \delta_{2}[y, z]_{b}-\delta_{1} \delta_{2}[y, z]_{c+}
\end{aligned}
$$

where $[y, z]_{x}=y(x) \overline{z^{\prime}(x)}-y^{\prime}(x) \overline{z(x)}(x \in I),[y, z]_{b}=$ $\lim _{x \rightarrow b}[y, z]_{x}$ exists and is finite.

Suppose that Weyl's limit circle case holds for the differential expression $l(y)$ on $I$. There are several sufficient conditions in which Weyl's limit circle case holds for a differential expression [21]. Denote by

$$
\theta(x)=\left\{\begin{array}{ll}
\theta_{1}(x), & x \in I_{1} \\
\theta_{2}(x), & x \in I_{2},
\end{array} \quad \varphi(x)= \begin{cases}\varphi_{1}(x), & x \in I_{1} \\
\varphi_{2}(x), & x \in I_{2}\end{cases}\right.
$$

the solutions of the equation $l(y)=\lambda y, x \in I$, satisfying the initial conditions

$$
\begin{array}{cc}
\theta_{1}(a, \lambda)=\cos \alpha, & \theta_{1}^{\prime}(a, \lambda)=\sin \alpha \\
\varphi_{1}(a, \lambda)=-\sin \alpha, & \varphi_{1}^{\prime}(a, \lambda)=\cos \alpha
\end{array}
$$

and transmission conditions

$$
\begin{array}{ll}
\theta_{2}(c+, \lambda)=\frac{\gamma_{1}}{\delta_{1}} \theta_{1}(c-, \lambda), & \theta_{2}^{\prime}(c+, \lambda)=\frac{\gamma_{2}}{\delta_{2}} \theta_{1}^{\prime}(c-, \lambda), \\
\varphi_{2}(c+, \lambda)=\frac{\gamma_{1}}{\delta_{1}} \varphi_{1}(c-, \lambda), & \varphi_{2}^{\prime}(c+, \lambda)=\frac{\gamma_{2}}{\delta_{2}} \varphi_{1}^{\prime}(c-, \lambda),
\end{array}
$$

where $\alpha, \gamma_{1}, \gamma_{2}, \delta_{1}$, and $\delta_{2} \in \mathbb{R}$ with $\gamma_{1} \gamma_{2}>0$ and $\delta_{1} \delta_{2}>$ 0 . The solutions $\theta(x, \lambda)$ and $\varphi(x, \lambda)$ belong to $H$. Let

$$
\begin{aligned}
& u(x)= \begin{cases}u_{1}(x)=\theta_{1}(x, 0), & x \in I_{1} \\
u_{2}(x)=\theta_{2}(x, 0), & x \in I_{2},\end{cases} \\
& v(x)= \begin{cases}v_{1}(x)=\varphi_{1}(x, 0), & x \in I_{1}, \\
v_{2}(x)=\varphi_{2}(x, 0), & x \in I_{2},\end{cases}
\end{aligned}
$$

$u(x)$, and $v(x)$ be solutions of the equation $l(y)=0, x \in I$, satisfying the initial conditions

$$
\begin{array}{ll}
u_{1}(a)=\cos \alpha, & u_{1}^{\prime}(a)=\sin \alpha, \\
v_{1}(a)=-\sin \alpha, & v_{1}^{\prime}(a)=\cos \alpha
\end{array}
$$

and transmission conditions

$$
\begin{array}{ll}
u_{2}(c+)=\frac{\gamma_{1}}{\delta_{1}} u_{1}(c-), & u_{2}^{\prime}(c+)=\frac{\gamma_{2}}{\delta_{2}} u_{1}^{\prime}(c-), \\
v_{2}(c+)=\frac{\gamma_{1}}{\delta_{1}} v_{1}(c-), & v_{2}^{\prime}(c+)=\frac{\gamma_{2}}{\delta_{2}} v_{1}^{\prime}(c-) .
\end{array}
$$

All the maximal dissipative extensions $\mathscr{L}_{G}$ of the operator $L_{0}$ are described by the following conditions (see $[4,15,18]$ ):

$$
\begin{gathered}
R_{1}(y): \gamma_{1} y(c-)-\delta_{1} y(c+)=0, \\
R_{2}(y): \gamma_{2} y^{\prime}(c-)-\delta_{2} y^{\prime}(c+)=0, \\
R_{3}(y): y(a) \cos \alpha+y^{\prime}(a) \sin \alpha=0, \\
R_{4}(y):[y, v]_{b}-G[y, u]_{b}=0, \quad \operatorname{Im} G>0 .
\end{gathered}
$$

Let us add the "incoming" and "outgoing" subspaces $D_{-}=$ $L^{2}(-\infty, 0)$ and $D_{+}=L^{2}(0, \infty)$ to $H$. The orthogonal sum $\bar{H}=$ $D_{-} \oplus H \oplus D_{+}$is called main Hilbert space of the dilation.

In the space $\mathscr{H}$, we consider the operator $\mathscr{L}_{G}$ on the set $D\left(\mathscr{L}_{G}\right)$, its elements consisting of vectors $w=\left\langle\varphi_{-}, \widehat{y}, \varphi_{+}\right\rangle$, generated by the expression

$$
\mathscr{L}_{G}\left\langle\varphi_{-}, \widehat{y}, \varphi_{+}\right\rangle=\left\langle i \frac{d \varphi_{-}}{d \xi}, l(\widehat{y}), i \frac{d \varphi_{+}}{d \xi}\right\rangle
$$

satisfying the conditions $\varphi_{-} \in W_{2}^{1}(-\infty, 0), \varphi_{+} \in W_{2}^{1}(0, \infty)$, $\hat{y} \in H, R_{1}(\hat{y})=0, R_{2}(\hat{y})=0, R_{3}(\hat{y})=0,[\hat{y}, v]_{b}-G[\hat{y}, u]_{b}=$ $\left(C / \sqrt{\delta_{1} \delta_{2}}\right) \varphi_{-}(0),[\hat{y}, v]_{b}-\bar{G}[\hat{y}, u]_{b}=\left(C / \sqrt{\delta_{1} \delta_{2}}\right) \varphi_{+}(0)$, where $W_{2}^{1}$ are Sobolev spaces and $C^{2}:=2 \operatorname{Im} G, C>0$.

Theorem 1. The operator $\mathscr{L}_{G}$ is self-adjoint in $\mathscr{H}$ and it is a self-adjoint dilation of the operator $\widetilde{L}_{G}\left(=L_{K}\right)$. 
Proof. We first prove that $\mathscr{L}_{\mathrm{G}}$ is symmetric in $\mathscr{H}$. Namely, $\left(\mathscr{L}_{G} f, g\right)_{\mathscr{H}}-\left(f, \mathscr{L}_{G} g\right)_{\mathscr{H}}=0$. Let $f, g \in D\left(\mathscr{L}_{G}\right), f=$ $\left\langle\varphi_{-}, \widehat{y}, \varphi_{+}\right\rangle$and $g=\left\langle\psi_{-}, \widehat{z}, \psi_{+}\right\rangle$. Then we have

$$
\begin{aligned}
\left(\mathscr{L}_{G} f, g\right)_{\mathscr{H}}-\left(f, \mathscr{L}_{G} g\right)_{\mathscr{H}} & \left(\mathscr{L}_{G}\left\langle\varphi_{-}, \widehat{y}, \varphi_{+}\right\rangle,\left\langle\psi_{-}, \widehat{z}, \psi_{+}\right\rangle\right) \\
& -\left(\left\langle\varphi_{-}, \widehat{y}, \varphi_{+}\right\rangle, \mathscr{L}_{G}\left\langle\psi_{-}, \widehat{z}, \psi_{+}\right\rangle\right) \\
= & \int_{-\infty}^{0} i \varphi_{-}^{\prime} \bar{\psi}_{-} d \xi+(l(\widehat{y}), \widehat{z})_{H}+\int_{0}^{\infty} i \varphi_{+}^{\prime} \bar{\psi}_{+} d \xi \\
& -\int_{-\infty}^{0} i \psi_{-}^{\prime} \bar{\varphi}_{-} d \xi-(\widehat{y}, l(\widehat{z}))_{H}-\int_{0}^{\infty} i \psi_{+}^{\prime} \bar{\varphi}_{+} d \xi \\
= & \int_{-\infty}^{0} i \varphi_{-}^{\prime} \bar{\psi}_{-} d \xi+\delta_{1} \delta_{2}\left[\widehat{y}, \widehat{z}_{b}+\int_{0}^{\infty} i \varphi_{+}^{\prime} \bar{\psi}_{+} d \xi\right. \\
& -\int_{-\infty}^{0} i \psi_{-}^{\prime} \bar{\varphi}_{-} d \xi-\int_{0}^{\infty} i \psi_{+}^{\prime} \bar{\varphi}_{+} d \xi \\
= & i \psi_{-}(0) \bar{\varphi}_{-}(0)-i \varphi_{+}(0) \bar{\psi}_{+}(0)+\delta_{1} \delta_{2}\left[\widehat{y}_{,}, \widehat{z}\right]_{b} .
\end{aligned}
$$

We obtain by direct computation

$$
i \psi_{-}(0) \bar{\varphi}_{-}(0)-i \varphi_{+}(0) \bar{\psi}_{+}(0)+\delta_{1} \delta_{2}[\widehat{y}, \widehat{z}]_{b}=0 .
$$

Thus, $\mathscr{L}_{G}$ is a symmetric operator. To prove that $\mathscr{L}_{G}$ is self-adjoint, we need to show that $\mathscr{L}_{G} \subseteq \mathscr{L}_{G}^{*}$. Take $g=$ $\left\langle\psi_{-}, \widehat{z}, \psi_{+}\right\rangle \in D\left(\mathscr{L}_{G}^{*}\right)$. Let $\mathscr{L}_{G}^{*} g=g^{*}=\left\langle\psi_{-}^{*}, \widehat{z}^{*}, \psi_{+}^{*}\right\rangle \in \mathscr{H}$, so that

$$
\left(\mathscr{L}_{G} f, g\right)_{\mathscr{H}}=\left(f, \mathscr{L}_{G}^{*} g\right)_{\mathscr{H}}=\left(f, g^{*}\right)_{\mathscr{H}}
$$

By choosing elements with suitable components as the $f \in D\left(\mathscr{L}_{G}\right)$ in (15), it is not difficult to show that $\psi_{-} \in$ $W_{2}^{1}(-\infty, 0), \psi_{+} \in W_{2}^{1}(0, \infty), g \in D(\mathscr{L})$, and $g^{*}=$ $\mathscr{L}_{G} g$; the operator $\mathscr{L}_{G}$ is defined by (12). Therefore (15) is obtained from $\left(\mathscr{L}_{G} f, g\right)_{\mathscr{H}}=\left(f, \mathscr{L}_{G} g\right)_{\mathscr{H}}$ for all $f \in$ $D\left(\mathscr{L}_{\mathrm{G}}^{*}\right)$. Furthermore, $g \in D\left(\mathscr{L}_{\mathrm{G}}^{*}\right)$ satisfies the conditions

$$
\begin{aligned}
{[\hat{y}, v]_{b}+G[\hat{y}, u]_{b} } & =\frac{C}{\sqrt{\delta_{1} \delta_{2}}} \varphi_{-}(0) \\
{[\hat{y}, v]_{b}+\bar{G}[\hat{y}, u]_{b} } & =\frac{C}{\sqrt{\delta_{1} \delta_{2}}} \varphi_{+}(0)
\end{aligned}
$$

Hence, $D\left(\mathscr{L}_{G}^{*}\right) \subseteq D\left(\mathscr{L}_{G}\right)$; that is, $\mathscr{L}_{G}=\mathscr{L}_{G}^{*}$.

The self-adjoint operator $\mathscr{L}_{G}$ generates on $\mathscr{H}$ a unitary group $U_{t}=\exp \left(i \mathscr{L}_{G} t\right)\left(t \in \mathbb{R}_{+}=(0, \infty)\right)$. Let us denote by $P: \mathscr{H} \rightarrow H$ and $P_{1}: H \rightarrow \mathscr{H}$ the mapping acting according to the formulae $P:\left\langle\varphi_{-}, \widehat{y}, \varphi_{+}\right\rangle \rightarrow \widehat{y}$ and $P_{1}:$ $\widehat{y} \rightarrow\langle 0, \hat{y}, 0\rangle$. Let $Z_{t}:=P U_{t} P_{1}, t \geq 0$, by using $U_{t}$. The family $\left\{Z_{t}\right\}(t \geq 0)$ of operators is a strongly continuous semigroup of completely nonunitary contraction on $H$. Let us denote by $B_{G}$ the generator of this semigroup: $B_{G} \hat{y}=$ $\lim _{t \rightarrow+0}(i t)^{-1}\left(Z_{t} \hat{y}-\widehat{y}\right)$. The domain of $B_{G}$ consists of all the vectors for which the limit exists. The operator $B_{G}$ is dissipative. The operator $\mathscr{L}_{G}$ is called the self-adjoint dilation of $B_{G}$ (see $\left.[10,21,30]\right)$. We show that $B_{G}=\widetilde{L}_{G}$; hence $\mathscr{L}_{G}$ is self-adjoint dilation of $B_{G}$. To show this, it is sufficient to verify the equality

$$
P\left(\mathscr{L}_{G}-\lambda I\right)^{-1} P_{1} \hat{y}=\left(\widetilde{L}_{G}-\lambda I\right)^{-1} \hat{y}, \quad \hat{y} \in H, \quad \operatorname{Im} h<0 .
$$

For this purpose, we set $\left(\mathscr{L}_{G}-\lambda I\right)^{-1} P_{1} \hat{y}=g=\left\langle\psi_{-}, \widehat{z}, \psi_{+}\right\rangle$ which implies that $\left(\mathscr{L}_{G}-\lambda I\right) g=P_{1} \widehat{y}$, and hence $\widetilde{l}(\widehat{z})-\lambda \widehat{z}=$ $\widehat{y}, \psi_{-}(\xi)=\psi_{-}(0) e^{-i \lambda \xi}$ and $\psi_{+}(\xi)=\psi_{+}(0) e^{-i \lambda \xi}$. Since $g \in$ $D\left(\mathscr{L}_{G}\right)$, then $\psi_{-} \in W_{2}^{1}(-\infty, 0)$; it follows that $\psi_{-}(0)=0$, and consequently $z$ satisfies the boundary condition $[\hat{y}, v]_{b}-$ $G[\widehat{y}, u]_{b}=0$. Therefore $\widehat{z} \in D\left(\widetilde{L}_{G}\right)$, and since point $\lambda$ with $\operatorname{Im} \lambda<0$ cannot be an eigenvalue of dissipative operator, then $\widehat{z}=\left(\widetilde{L}_{G}-\lambda I\right)^{-1} \widehat{y}$. Thus we have

$$
\begin{aligned}
& \left(\mathscr{L}_{G}-\lambda I\right)^{-1} P_{1} \hat{y} \\
& \quad=\left\langle 0,\left(\widetilde{L}_{G}-\lambda I\right)^{-1} \hat{y}, \frac{\sqrt{\delta_{1} \delta_{2}}}{C}\left([\hat{y}, v]_{b}+\bar{G}[\hat{y}, u]_{b}\right) e^{-i \lambda \xi}\right\rangle
\end{aligned}
$$

for $\hat{y}$ and $\operatorname{Im} \lambda<0$. By applying onto the mapping $P$, we obtain (17), and

$$
\begin{aligned}
\left(\widetilde{L}_{G}-\lambda I\right)^{-1} & =P\left(\mathscr{L}_{G}-\lambda I\right)^{-1} P_{1}=-i P \int_{0}^{\infty} U_{t} e^{-i \lambda t} d t P_{1} \\
& =-i \int_{0}^{\infty} Z_{t} e^{-i \lambda t} d t=\left(B_{G}-\lambda I\right)^{-1}, \quad \operatorname{Im} \lambda<0,
\end{aligned}
$$

so this clearly shows that $\widetilde{L}_{G}=B_{G}$.

\section{Functional Model of Dissipative Sturm-Liouville Operator}

The unitary group $\left\{U_{t}\right\}$ has an important property which makes it possible to apply it to the Lax-Phillips [23]. It has orthogonal incoming and outgoing subspaces $D_{-}=$ $\left\langle L^{2}(-\infty, 0), 0,0\right\rangle$ and $D_{+}=\left\langle 0,0, L^{2}(0, \infty)\right\rangle$ having the following properties:

(1) $U_{t} D_{-} \subset D_{-}, t \leq 0$ and $U_{t} D_{+} \subset D_{+}, t \geq 0$,

(2) $\cap_{t \leq 0} U_{t} D_{-}=\cap_{t \geq 0} U_{t} D_{+}=\{0\}$,

(3) $\overline{U_{t \geq 0} U_{t} D_{-}}=\overline{U_{t \leq 0} U_{t} D_{+}}=\mathscr{H}$,

(4) $D_{-} \perp D_{+}$.

Property (4) is clear. To be able to prove property (1) for $D_{+}$(the proof for $D_{-}$is similar), we set $\mathscr{R}_{\lambda}=\left(\mathscr{L}_{G}-\lambda I\right)^{-1}$. For all $\lambda$, with $\operatorname{Im} \lambda<0$ and for any $f=\left\langle 0,0, \varphi_{+}\right\rangle \in D_{+}$, we have

$$
\mathscr{R}_{\lambda} f=\left\langle 0,0,-i e^{-i \lambda \xi} \int_{0}^{\xi} e^{i \lambda s} \varphi_{+}(s) d s\right\rangle,
$$

as $\mathscr{R}_{\lambda} f \in D_{+}$. Therefore, if $g \perp D_{+}$, then

$$
0=\left(\mathscr{R}_{\lambda} f, g\right)_{\mathscr{H}}=-i \int_{0}^{\infty} e^{-i \lambda t}\left(U_{t} f, g\right)_{\mathscr{H}} d t, \quad \operatorname{Im} \lambda<0,
$$


which implies that $\left(U_{t} f, g\right)_{\mathscr{H}}=0$ for all $t \geq 0$. Hence, for $t \geq 0, U_{t} D_{+} \subset D_{+}$, and property (1) has been proved.

In order to prove property (2), we define the mappings $P^{+}: \mathscr{H} \rightarrow L^{2}(0, \infty)$ and $P_{1}^{+}: L^{2}(0, \infty) \rightarrow D_{+}$as follows: $P^{+}:\left\langle\varphi_{-}, \widehat{y}, \varphi_{+}\right\rangle \rightarrow \varphi_{+}$and $P_{1}^{+}: \varphi \rightarrow\langle 0,0, \varphi\rangle$, respectively. We take into consider that the semigroup of isometries $U_{t}^{+}:=P^{+} U_{t} P_{1}^{+}(t \geq 0)$ is a one-sided shift in $L^{2}(0, \infty)$. Indeed, the generator of the semigroup of the onesided shift $V_{t}$ in $L^{2}(0, \infty)$ is the differential operator $i(d / d \xi)$ with the boundary condition $\varphi(0)=0$. On the other hand, the generator $S$ of the semigroup of isometries $U_{t}^{+}(t \geq$ $0)$ is the operator $S \varphi=P^{+} \mathscr{L}_{G} P_{1}^{+} \varphi=P^{+} \mathscr{L}_{G}\langle 0,0, \varphi\rangle=$ $P^{+}\langle 0,0, i(d / d \xi) \varphi\rangle=i(d / d \xi) \varphi$, where $\varphi \in W_{2}^{1}(0, \infty)$ and $\varphi(0)=0$. Since a semigroup is uniquely determined by its generator, it follows that $U_{t}^{+}=V_{t}$, and, hence,

$$
\bigcap_{t \geq 0} U_{t} D_{+}=\left\langle 0,0, \bigcap_{t \leq 0} V_{t} L^{2}(0, \infty)\right\rangle=\{0\},
$$

so the proof is completed.

Definition 2. The linear operator $A$ with domain $D(A)$ acting in the Hilbert space $H$ is called completely non-self-adjoint (or simple) if there is no invariant subspace $M \subseteq D(A)(M \neq\{0\})$ of the operator $A$ on which the restriction $A$ to $M$ is selfadjoint.

To prove property (3) of the incoming and outgoing subspaces, let us prove following lemma.

Lemma 3. The operator $\widetilde{L}_{G}$ is completely non-self-adjoint (simple).

Proof. Let $H^{\prime} \subset H$ be a nontrivial subspace in which $\widetilde{L}_{G}$ induces a self-adjoint operator $\widetilde{L}_{G}^{\prime}$ with domain $D\left(\widetilde{L}_{G}^{\prime}\right)=$ $H^{\prime} \cap D\left(\widetilde{L}_{G}\right)$. If $\widehat{f} \in D\left(\widetilde{L}_{G}^{\prime}\right)$, then $\widehat{f} \in D\left(\widetilde{L}_{G}^{*}\right)$ and

$$
\begin{aligned}
0 & =\frac{d}{d t}\left\|e^{i \widetilde{L}_{G}^{\prime} t} \widehat{f}\right\|_{H}^{2}=\frac{d}{d t}\left(e^{i \widetilde{L}_{G}^{\prime} t} \widehat{f}, e^{i \widetilde{L}_{G}^{\prime} t} \widehat{f}\right)_{H} \\
& =-C^{2}\left(\left[e^{i \widetilde{L}_{G}^{\prime} t} \widehat{f}, u\right]_{b}\right)_{H}^{2} \cdot
\end{aligned}
$$

Consequently, we have $[\hat{y}, u]_{b}=0$. Using this result with boundary condition $[\hat{y}, v]_{b}+G[\hat{y}, u]_{b}=0$, we have $[\hat{y}, u]_{b}=$ 0 ; that is, $\hat{y}(\lambda)=0$. Since all solutions of $l(y)=\lambda y$ belong to $L^{2}(0, \infty)$, from this it can be concluded that the resolvent $R_{\lambda}\left(\widetilde{L}_{G}\right)$ is a compact operator, and the spectrum of $\widetilde{L}_{G}$ is purely discrete. Consequently, by the theorem on expansion in the eigenvectors of the self-adjoint operator $\widetilde{L}_{G}^{\prime}$ we obtain $H^{\prime}=\{0\}$. Hence the operator $\widetilde{L}_{G}$ is simple. The proof is completed.

$$
\text { Let us define } H_{-}=\overline{U_{t \geq 0} U_{t} D_{-}}, H_{+}=\overline{U_{t \leq 0} U_{t} D_{+}} \text {. }
$$

Lemma 4. The equality $H_{-}+H_{+}=\mathscr{H}$ holds.

Proof. Considering property (1) of the subspace $D_{+}$, it is easy to show that the subspace $\mathscr{H}^{\prime}=\mathscr{H} \odot\left(H_{-}+H_{+}\right)$is invariant relative to the group $\left\{U_{t}\right\}$ and has the form $\mathscr{H}^{\prime}=\left\langle 0, H^{\prime}, 0\right\rangle$, where $H^{\prime}$ is a subspace in $H$. Therefore, if the subspace $\mathscr{H}^{\prime}$ (and hence also $H^{\prime}$ ) was nontrivial, then the unitary group $\left\{U_{t}^{\prime}\right\}$ restricted to this subspace would be a unitary part of the group $\left\{U_{t}\right\}$, and hence, the restriction $\widetilde{L}_{G}^{\prime}$ of $\widetilde{L}_{G}$ to $H^{\prime}$ would be a self-adjoint operator in $H^{\prime}$. Since the operator $\widetilde{L}_{G}$ is simple, it follows that $H^{\prime}=\{0\}$. The lemma is proved.

Assume that

$$
\begin{aligned}
& \varphi(x, \lambda):= \begin{cases}\varphi_{1}(x, \lambda), & x \in I_{1} \\
\varphi_{2}(x, \lambda), & x \in I_{2},\end{cases} \\
& \psi(x, \lambda):= \begin{cases}\psi_{1}(x, \lambda), & x \in I_{1} \\
\psi_{2}(x, \lambda), & x \in I_{2}\end{cases}
\end{aligned}
$$

are solutions of $l(y)=\lambda y$ satisfying the conditions

$$
\begin{aligned}
\varphi_{1}(a, \lambda)=\cos \alpha, & \varphi_{1}^{\prime}(a, \lambda)=\sin \alpha, \\
\psi_{1}(a, \lambda)=-\sin \alpha, & \psi_{1}^{\prime}(a, \lambda)=\cos \alpha, \\
\varphi_{2}(c+, \lambda)=\frac{\gamma_{1}}{\delta_{1}} \varphi_{1}(c-, \lambda), & \varphi_{2}^{\prime}(c+, \lambda)=\frac{\gamma_{2}}{\delta_{2}} \varphi_{1}^{\prime}(c-, \lambda) .
\end{aligned}
$$

Let us adopt the following notations:

$$
\begin{gathered}
K(\lambda)=\frac{[\varphi, v]_{b}}{[\psi, u]_{b}}, \quad M(\lambda)=-\frac{[\psi, u]_{b}}{[\varphi, u]_{b}}, \\
S_{G}(\lambda)=(M(\lambda) K(\lambda)+G)(M(\lambda) K(\lambda)+\bar{G})^{-1},
\end{gathered}
$$

where $M(\lambda)$ is a meromorphic function on the complex plane $\mathbb{C}$ with a countable number of poles on the real axis. Further, it is possible to show that the function $M(\lambda)$ possesses the following properties: $\operatorname{Im} M(\lambda) \leq 0$ for all $\operatorname{Im} \lambda \neq 0$, and $\bar{M}(\lambda)=M(\bar{\lambda})$ for all $\lambda \in \mathbb{C}$, except the real poles $M(\lambda)$.

We set

$$
\begin{aligned}
& U_{\lambda}^{-}(x, \xi, \zeta) \\
& =\left\langle e^{-i \lambda \xi},\right. \\
& \quad \frac{C}{\sqrt{\delta_{1} \delta_{2}}} M(\lambda)\left[(M(\lambda) K(\lambda)+G)[\psi, u]_{b}\right]^{-1} \varphi(x, \lambda), \\
& \left.\quad \overline{S_{G}}(\lambda) e^{-i \lambda \zeta}\right\rangle .
\end{aligned}
$$

We note that the vectors $U_{\lambda}^{-}(x, \xi, \zeta)$ for real $\lambda$ do not belong to the space $\mathscr{H}$. However, $U_{\lambda}^{-}(x, \xi, \zeta)$ satisfies the equation $\mathscr{L} U=\lambda U$ and the corresponding boundary conditions for the operator $\mathscr{L}_{h}$.

By means of vector $U_{\lambda}^{-}(x, \xi, \zeta)$, we define the transformation $F_{-}: f \rightarrow \widetilde{f_{-}}(\lambda)$ by

$$
\left(F_{-} f\right)(\lambda):=\widetilde{f_{-}}(\lambda):=\frac{1}{\sqrt{2 \pi}}(f, U \bar{\lambda})_{\mathscr{H}}
$$


on the vectors $f=\left\langle\varphi_{-}, \widehat{y}, \varphi_{+}\right\rangle$in which $\varphi_{-}(\xi), \varphi_{+}(\zeta), y(x)$ are smooth, compactly supported functions.

Lemma 5. The transformation $F_{-}$isometrically maps $H_{-}$onto $L^{2}(\mathbb{R})$. For all vectors $f, g \in H_{-}$the Parseval equality and the inversion formulae hold:

$$
\begin{gathered}
(f, g)_{\mathscr{H}}=\left(\widetilde{f_{-}}, \widetilde{g_{-}}\right)_{L^{2}}=\int_{-\infty}^{\infty} \widetilde{f_{-}}(\lambda) \overline{\widetilde{g_{-}}}(\lambda) d \lambda, \\
f=\frac{1}{\sqrt{2 \pi}} \int_{-\infty}^{\infty} \widetilde{f_{-}}(\lambda) U_{\bar{\lambda}} d \lambda,
\end{gathered}
$$

where $\widetilde{f_{-}}(\lambda)=\left(F_{-} f\right)(\lambda)$ and $\widetilde{g_{-}}(\lambda)=\left(F_{-} g\right)(\lambda)$.

Proof. For $f, g \in D_{-}, f=\left\langle\varphi_{-}, 0,0\right\rangle, g=\left\langle\psi_{-}, 0,0\right\rangle$, with Paley-Wiener theorem, we have

$$
\widetilde{f_{-}}(\lambda)=\frac{1}{\sqrt{2 \pi}}\left(f, U_{\bar{\lambda}}\right)_{\mathscr{H}}=\frac{1}{\sqrt{2 \pi}} \int_{-\infty}^{0} \varphi_{-}(\xi) e^{-i \lambda \xi} d \xi \in H_{-}^{2}
$$

and by using usual Parseval equality for Fourier integrals,

$$
\begin{aligned}
(f, g)_{\mathscr{H}} & =\int_{-\infty}^{\infty} \varphi_{-}(\xi) \overline{\psi_{-}(\xi)} d \xi \\
& =\int_{-\infty}^{\infty} \widetilde{f_{-}}(\lambda) \overline{\widetilde{g_{-}}(\lambda)} d \lambda=\left(F_{-} f, F_{-} g\right)_{L^{2}} .
\end{aligned}
$$

Here, $H_{ \pm}^{2}$ denote the Hardy classes in $L^{2}(\mathbb{R})$ consisting of the functions analytically extendible to the upper and lower halfplanes, respectively.

We now extend the Parseval equality to the whole of $H_{-}$. We consider in $H_{-}$the dense set of $H_{-}^{\prime}$ of the vectors obtained as follows from the smooth, compactly supported functions in $D_{-}: f \in H_{-}^{\prime}$ if $f=U_{T} f_{0}, f_{0}=\left\langle\varphi_{-}, 0,0\right\rangle, \varphi_{-} \in C_{0}^{\infty}(-\infty, 0)$, where $T=T_{f}$ is a nonnegative number depending on $f$. If $f, g \in H_{-}^{\prime}$, then for $T>T_{f}$ and $T>T_{g}$ we have $U_{-T} f, U_{-T} g \in$ $D_{-}$; moreover, the first components of these vectors belong to $C_{0}^{\infty}(-\infty, 0)$. Therefore, since the operators $U_{t}(t \in \mathbb{R})$ are unitary, by the equality

$$
F_{-} U_{t} f=\left(U_{t} f, U_{\lambda}^{-}\right)_{\mathscr{H}}=e^{i \lambda t}\left(f, U_{\lambda}^{-}\right)_{\mathscr{H}}=e^{i \lambda t} F_{-} f,
$$

we have

$$
\begin{aligned}
(f, g)_{\mathscr{H}}= & \left(U_{-T} f, U_{-T} g\right)_{\mathscr{H}}=\left(F_{-} U_{-T} f, F_{-} U_{-T} g\right)_{L^{2}}, \\
& \left(e^{i \lambda T} F_{-} f, e^{i \lambda T} F_{-} g\right)_{L^{2}}=(\tilde{f}, \tilde{g})_{L^{2}} .
\end{aligned}
$$

By taking the closure (35), we obtain the Parseval equality for the space $H_{-}$. The inversion formula is obtained from the Parseval equality if all integrals in it are considered as limits in the integrals over finite intervals. Finally $F_{-} H_{-}=$ $\overline{\cup_{t \geq 0} F_{-} U_{t} D_{-}}=\overline{U_{t \geq 0} e^{i \lambda t} H_{-}^{2}}=L^{2}(\mathbb{R})$; that is, $F_{-}$maps $H_{-}$onto the whole of $L^{2}(\mathbb{R})$. The lemma is proved.
We set

$$
\begin{aligned}
& U_{\lambda}^{+}(x, \xi, \zeta) \\
& =\left\langle S_{G}(\lambda) e^{-i \lambda \xi},\right. \\
& \quad \frac{C}{\sqrt{\delta_{1} \delta_{2}}} M(\lambda)\left[(M(\lambda) K(\lambda)+\bar{G})[\psi, u]_{b}\right]^{-1} \varphi(x, \lambda), \\
& \left.e^{-i \lambda \zeta}\right\rangle .
\end{aligned}
$$

We note that the vectors $U_{\lambda}^{+}(x, \xi, \zeta)$ for real $\lambda$ do not belong to the space $\mathscr{H}$. However, $U_{\lambda}^{+}(x, \xi, \zeta)$ satisfies the equation $\mathscr{L} U=\lambda U$ and the corresponding boundary conditions for the operator $\mathscr{L}_{h}$. With the help of vector $U_{\lambda}^{+}(x, \xi, \zeta)$, we define the transformation $F_{+}: f \rightarrow \widetilde{f_{+}}(\lambda)$ by $\left(F_{+} f\right)(\lambda):=\widetilde{f_{+}}(\lambda):=$ $(1 / \sqrt{2 \pi})\left(f, U_{\lambda}^{+}\right)_{\mathscr{H}}$ on the vectors $f=\left\langle\varphi_{-}, \hat{y}, \varphi_{+}\right\rangle$in which $\varphi_{-}(\xi), \varphi_{+}(\zeta)$, and $y(x)$ are smooth, compactly supported functions.

Lemma 6. The transformation $F_{+}$isometrically maps $H_{+}$onto $L^{2}(\mathbb{R})$. For all vectors $f, g \in H_{+}$the Parseval equality and the inversion formula hold:

$$
\begin{gathered}
(f, g)_{\mathscr{H}}=\left(\widetilde{f_{+}}, \widetilde{g_{+}}\right)_{L^{2}}=\int_{-\infty}^{\infty} \widetilde{f_{+}}(\lambda) \overline{\widetilde{g_{+}}(\lambda)} d \lambda, \\
f=\frac{1}{\sqrt{2 \pi}} \int_{-\infty}^{\infty} \widetilde{f_{+}}(\lambda) U_{\lambda}^{+} d \lambda,
\end{gathered}
$$

where $\widetilde{f_{+}}(\lambda)=\left(F_{+} f\right)(\lambda)$ and $\widetilde{g_{+}}(\lambda)=\left(F_{+} g\right)(\lambda)$.

Proof. The proof is analogous to Lemma 6.

It is obvious that the matrix-valued function $S_{G}(\lambda)$ is meromorphic in $\mathbb{C}$ and all poles are in the lower half-plane. From (27), $\left|S_{G}(\lambda)\right| \leq 1$ for $\operatorname{Im} \lambda>0$, and $S_{G}(\lambda)$ is the unitary matrix for all $\lambda \in \mathbb{R}$. Therefore, it explicitly follows from the formulae for the vectors $U_{\lambda}^{-}$and $U_{\lambda}^{+}$that

$$
U_{\lambda}^{+}=S_{G}(\lambda) U_{\lambda}^{-} .
$$

It follows from Lemmas 6 and 5 that $H_{-}=H_{+}$. Together with Lemma 5, this shows that $H_{-}=H_{+}=\mathscr{H}$; therefore property (3) has been proved for the incoming and outgoing subspaces. Thus, the transformation $F_{-}$isometrically maps $H_{-}$onto $L^{2}(\mathbb{R})$ with the subspace $D_{-}$mapped onto $H_{-}^{2}$ and the operators $U_{t}$ are transformed into the operators of multiplication by $e^{i \lambda t}$. This means that $F_{-}$is the incoming spectral representation for the group $\left\{U_{t}\right\}$. Similarly, $F_{+}$is the outgoing spectral representation for the group $\left\{U_{t}\right\}$. It follows from (38) that the passage from the $F_{-}$representation of an element $f \in \mathscr{H}$ to its $F_{+}$representation is accomplished as $\widetilde{f_{+}}(\lambda)=$ $S_{G}^{-1}(\lambda) \widetilde{f_{-}}(\lambda)$. Consequently, according to [22], we have proved the following.

Theorem 7. The function $\overline{S_{G}}(\lambda)$ is the scattering matrix of the group $\left\{U_{t}\right\}$ (of the self-adjoint operator $\mathscr{L}_{G}$ ). 
Let $S(\lambda)$ be an arbitrary nonconstant inner function (see [19]) on the upper half-plane (the analytic function $S(\lambda)$ and the upper half-plane $\mathbb{C}_{+}$is called inner function on $\mathbb{C}_{+}$if $\left|S_{h}(\lambda)\right| \leq 1$ for all $\lambda \in \mathbb{C}_{+}$and $\left|S_{h}(\lambda)\right|=1$ for almost all $\lambda \in \mathbb{R}$ ). Define $K=H_{+}^{2} \odot S H_{+}^{2}$. Then $K \neq\{0\}$ is a subspace of the Hilbert space $H_{+}^{2}$. We consider the semigroup of operators $Z_{t}(t \geq 0)$ acting in $K$ according to the formula $Z_{t} \varphi=P\left[e^{i \lambda t} \varphi\right], \varphi=\varphi(\lambda) \in K$, where $P$ is the orthogonal projection from $H_{+}^{2}$ onto $K$. The generator of the semigroup $\left\{Z_{t}\right\}$ is denoted by

$$
T \varphi=\lim _{t \rightarrow+0}(i t)^{-1}\left(Z_{t} \varphi-\varphi\right),
$$

where $T$ is a maximal dissipative operator acting in $K$ and with the domain $D(T)$ consisting of all functions $\varphi \in$ $K$, such that the limit exists. The operator $T$ is called $a$ model dissipative operator. Recall that this model dissipative operator, which is associated with the names of Lax-Phillips [23], is a special case of a more general model dissipative operator constructed by Nagy and Foiaş [22]. The basic assertion is that $S(\lambda)$ is the characteristic function of the operator $T$.

Let $K=\langle 0, H, 0\rangle$, so that $\mathscr{H}=D_{-} \oplus K \oplus D_{+}$. It follows from the explicit form of the unitary transformation $F_{-}$under the mapping $F_{-}$that

$$
\begin{gathered}
\mathscr{H} \longrightarrow L^{2}(\mathbb{R}), \quad f \longrightarrow \widetilde{f_{-}}(\lambda)=\left(F_{-} f\right)(\lambda), \\
D_{-} \longrightarrow H_{-}^{2}, \quad D_{+} \longrightarrow S_{G} H_{+}^{2}, \\
K \longrightarrow H_{+}^{2} \Theta S_{G} H_{+}^{2}, \\
U_{t} \longrightarrow\left(F_{-} U_{t} F_{-}^{-1} \widetilde{f_{-}}\right)(\lambda)=e^{i \lambda t} \widetilde{f_{-}}(\lambda) .
\end{gathered}
$$

The formulas (40) show that operator $\widetilde{L}_{G}$ is unitarily equivalent to the model dissipative operator with the characteristic function $S_{G}(\lambda)$. We have thus proved the following theorem.

Theorem 8. The characteristic function of the maximal dissipative operator $\widetilde{L}_{G}$ coincides with the function $S_{G}(\lambda)$ defined by (27).

\section{The Spectral Properties of Dissipative Sturm-Liouville Operators}

By using characteristic function, the spectral properties of the maximal dissipative operator $\widetilde{L}_{G}\left(L_{K}\right)$ can be investigated. The characteristic function of the maximal dissipative operator $\widetilde{L}_{G}$ is known to lead to information of completeness about the spectral properties of this operator. For instance, the absence of a singular factor $s(\lambda)$ of the characteristic function $S_{G}(\lambda)$ in the factorization $\operatorname{det} S_{G}(\lambda)=s(\lambda) B(\lambda)(B(\lambda)$ is a Blaschke product) ensures completeness of the system of eigenvectors and associated vectors of the operator $\widetilde{L}_{G}\left(L_{K}\right)$ in the space $L_{2}(0, \infty)$ (see $\left.[10,21,30]\right)$. If the characteristic function $S_{G}(\lambda)$ has nontrivial singular factor, the system of eigenvectors and associated vectors of the operator $\widetilde{L}_{G}\left(L_{K}\right)$ can fail to be complete. Because $S_{G}(\lambda)$ is smooth, the support of the corresponding singular measure $\mu$ must be contained in the set of poles $S_{G}(\lambda)$. But in this case the singular measure $\mu$ is a simple step function. If we require $S_{G}(\lambda)$ to have no zeros of infinite multiplicity, then $\mu=0$. So the singular factor vanishes. The characteristic function $S_{G}(\lambda)$ of the maximal dissipative operator $\widetilde{L}_{G}$ has the form

$$
S_{G}(\lambda):=\frac{M(\lambda) K(\lambda)+G}{M(\lambda) K(\lambda)+\bar{G}},
$$

where $\operatorname{Im} G>0$.

Theorem 9. For all the values of $G$ with $\operatorname{Im} G>0$, except possibly for a single value $G=G_{0}$, the characteristic function $S_{G}(\lambda)$ of the maximal dissipative operator $\widetilde{L}_{G}$ is a Blaschke product. The spectrum of $\widetilde{L}_{G}$ is purely discrete and belongs to the open upper half-plane. The operator $\widetilde{L}_{G}\left(G \neq G_{0}\right)$ has a countable number of isolated eigenvalues with finite multiplicity and limit points at infinity. The system of all eigenvectors and associated vectors of the operator $\widetilde{L}_{G}$ is complete in the space $H$.

Proof. From (35), it is clear that $S_{G}(\lambda)$ is an inner function in the upper half-plane, and it is meromorphic in the whole complex $\lambda$-plane. Therefore, it can be factored in the form

$$
S_{G}(\lambda)=e^{i \lambda c} B_{G}(\lambda), \quad c=c(G) \geq 0,
$$

where $B_{G}(\lambda)$ is a Blaschke product. It follows from (42) that

$$
\left|S_{G}(\lambda)\right|=\left|e^{i \lambda c}\right|\left|B_{G}(\lambda)\right| \leq e^{-b(G) \operatorname{Im} \lambda}, \quad \operatorname{Im} \lambda \geq 0 .
$$

Further, expressing $n_{G}(\lambda):=M(\lambda) K(\lambda)$ in terms of $S_{G}(\lambda)$, we find from (35) that

$$
n_{G}(\lambda)=\frac{\bar{G} S_{G}(\lambda)-G}{1-S_{G}(\lambda)} .
$$

For a given value $G(\operatorname{Im} G>0)$, if $c(G)>0$, then (43) implies that $\lim _{t \rightarrow+\infty} S_{G}(i t)=0$, and then (44) gives us that $\lim _{t \rightarrow+\infty} n_{G}(i t)=G_{0}$. Since $n_{G}(\lambda)$ does not depend on $G$, this implies that $c(G)$ can be nonzero at not more than a single point $G=G_{0}$ (and further $G_{0}=-\lim _{t \rightarrow+\infty} n_{G}(i t)$ ). This completes the proof.

\section{References}

[1] C. T. Fulton, "Two-point boundary value problems with eigenvalue parameter contained in the boundary conditions," Proceedings of the Royal Society of Edinburgh A, vol. 77, no. 3-4, pp. 293-308, 1977.

[2] C. T. Fulton, "Singular eigenvalue problems with eigenvalue parameter contained in the boundary conditions," Proceedings of the Royal Society of Edinburgh A, vol. 87, no. 1-2, pp. 1-34, 1980.

[3] I. C. Gohberg and M. G. Krein, Introduction to the Theory of Linear Nonselfadjoint Operators, American Mathematical Society, Providence, RI, USA, 1969.

[4] O. S. Mukhtarov and M. Kadakal, "Some spectral properties of one Sturm-Liouville type problem with discontinuous weight," Siberian Mathematical Journal, vol. 46, no. 4, pp. 681-694, 2005. 
[5] B. P. Allahverdiev, "On dilation theory and spectral analysis of dissipative Schrödinger operators in Weyl's limit-circle case," Izvestiya Akademii Nauk, vol. 54, pp. 242-257, 1990, English translation: Mathematics of the USSR-Izvestiya, vol. 36, pp. 247262, 1991.

[6] A. A. Shkalikov, "Boundary value problems with a spectral parameter in the boundary conditions," Zeitschrift Für Angewandte Mathematik Und Mechanik, vol. 76, pp. 133-135, 1996.

[7] S. Saltan and B. P. Allahverdiev, "Spectral analysis of nonselfadjoint Schrödinger operators with a matrix potential," Journal of Mathematical Analysis and Applications, vol. 303, no. 1, pp. 208219, 2005.

[8] B. P. Allahverdiev, "A nonself-adjoint singular Sturm-Liouville problem with a spectral parameter in the boundary condition," Mathematische Nachrichten, vol. 278, no. 7-8, pp. 743-755, 2005.

[9] B. P. Allahverdiev, "Spectral analysis of dissipative Dirac operators with general boundary conditions," Journal of Mathematical Analysis and Applications, vol. 283, no. 1, pp. 287-303, 2003.

[10] B. P. Allahverdiev, "Dilation and functional model of dissipative operator generated by an infinite Jacobi matrix," Mathematical and Computer Modelling, vol. 38, no. 10, pp. 989-1001, 2003.

[11] M. Y. Ongun and B. P. Allahverdiev, "A completeness theorem for a dissipative Schrödinger problem with the spectral parameter in the boundary condition," Mathematische Nachrichten, vol. 281, no. 4, pp. 541-554, 2008.

[12] A. Ery1lmaz, "Spectral analysis of $q$-sturm-liouville problem with the spectral parameter in the boundary condition," Journal of Function Spaces and Applications, vol. 2012, Article ID 736437, 17 pages, 2012.

[13] H. Tuna, "Dissipative $q$-difference operatorsof the SturmLiouville type," Applications of Mathematics. In press.

[14] H. Tuna, "Functionalmodel of dissipative fourth order differential operators," Analele Stiintice ale Universitatii Ovidius Constanta. In press.

[15] E. Bairamov and E. Ugurlu, "The determinants of dissipative Sturm-Liouville operators with transmission conditions," Mathematical and Computer Modelling, vol. 53, no. 5-6, pp. 805-813, 2011.

[16] O. Mukhtarov, "Discontinuous boundary value problem with spectral parameter in boundary conditions," Turkish Journal of Mathematics, vol. 18, no. 2, pp. 183-192, 1994.

[17] O. S. Mukhtarov and E. Tunç, "Eigenvalue problems for SturmLiouville equations with transmission conditions," Israel Journal of Mathematics, vol. 144, pp. 367-380, 2004.

[18] Z. Akdoğan, M. Demirci, and O. S. Mukhtarov, "Green function of discontinuous boundary value problem with transmission conditions," Mathematical Methods in the Applied Sciences, vol. 30, pp. 1719-1738, 2007.

[19] O. S. Muhtarov and S. Yakubov, "Problems for ordinary differential equations with transmission conditions," Applicable Analysis, vol. 81, no. 5, pp. 1033-1064, 2002.

[20] A. Wang, J. Sun, X. Hao, and S. Yao, "Completeness of eigenfunctions of Sturm-Liouville problems with transmission conditions," Methods and Applications of Analysis, vol. 16, no. 3, pp. 299-312, 2009.

[21] M. A. Naimark, Lineinye Differentsialnye Operatory, Nauka, Moscow, Russia, 2nd edition, 1969, English Translation of 1st ed., vol. 1-2, Ungar, New York, NY, USA, 1967, 1968.

[22] B. S. Nagy and C. Foiaş, Analyse Harmonique Des Operateurs De L.Espace De Hilbert, Masson, Paris, France, 1967, English Translation North-Holland, Amsterdam, The Netherlands, 1970.
[23] P. D. Lax and R. S. Phillips, Scattering Theory, Academic Press, New York, Ny, USA, 1967.

[24] B. S. Pavlov, "Dilation theory and spectral analysis of nonselfadjoint differential operators," in Mathematical Programming and Related Questions (Proc. Seventh Winter School, Drogobych, 1974), pp. 3-69, Central Economic Mathematical Institute, Moscow, Russia, 1974.

[25] B. S. Pavlov, "Dilation theoryand spectral analysis of nonselfadjoint differential operators," American Mathematical Society Translations, vol. 115, pp. 103-142, 1981.

[26] B. S. Pavlov, "Self-adjoint dilatation of a dissipative Schrödinger operator and an eigenfunction expansion," Functional Analysis and Its Applications, vol. 9, no. 2, pp. 172-173, 1975.

[27] B. S. Pavlov, "Selfadjoint dilation of a dissipative schrödinger operator and eigenfunction expansion," Functional Analysis and Its Applications, vol. 98, pp. 172-173, 1975.

[28] B. S. Pavlov, "Selfadjoint dilation of a dissipative Schrödinger operator and its resolution in terms of eigenfunctions," Mathematics of the USSR-Sbornik, vol. 31, no. 4, pp. 457-478, 1977.

[29] A. Kuzhel, Characteristic Functions and Models of Nonselfadjoint Operators, vol. 349, Kluwer Academic, Dordrecht, The Netherlands, 1996.

[30] S. Kuzhel, "On an inverse problem in the Lax-Phillips scattering scheme for a class of operator-differential equations," St. Petersburg Mathematical Journal, vol. 13, no. 1, pp. 60-83, 2001. 


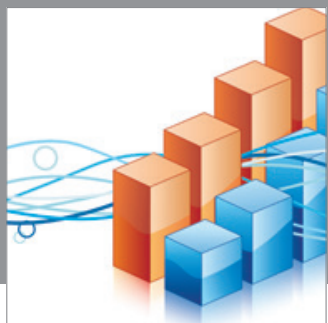

Advances in

Operations Research

mansans

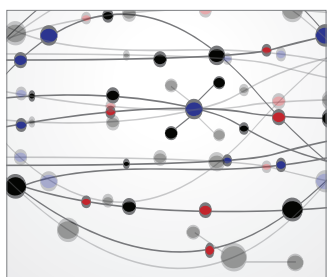

The Scientific World Journal
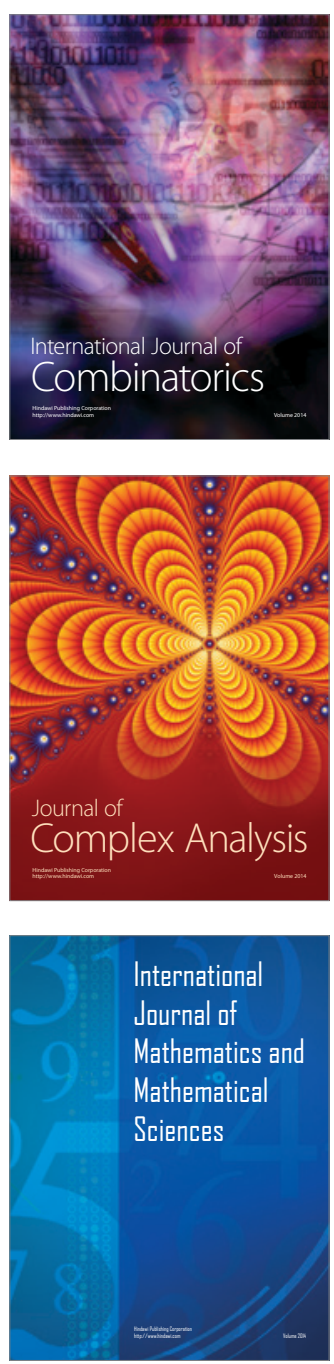
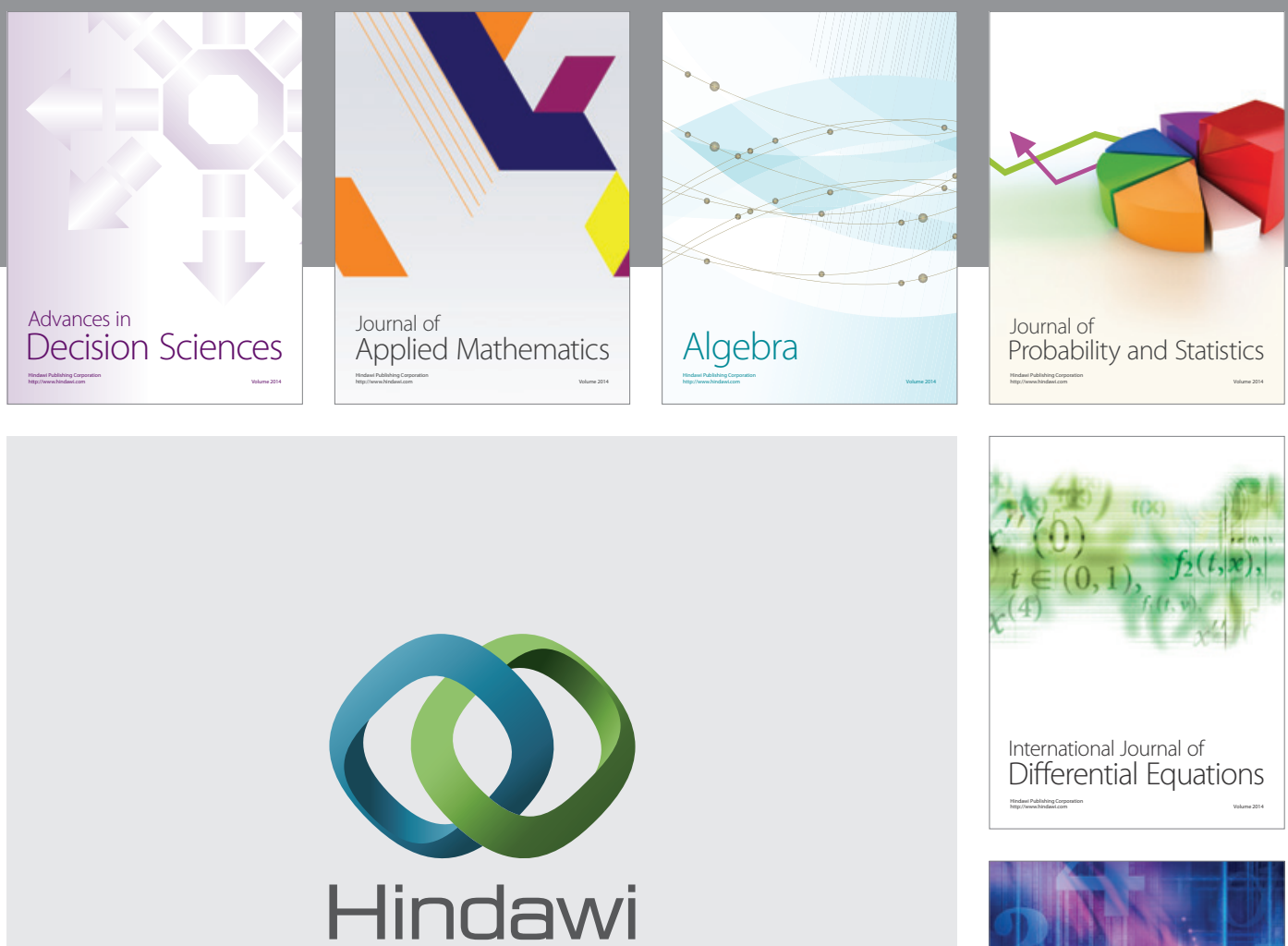

Submit your manuscripts at http://www.hindawi.com
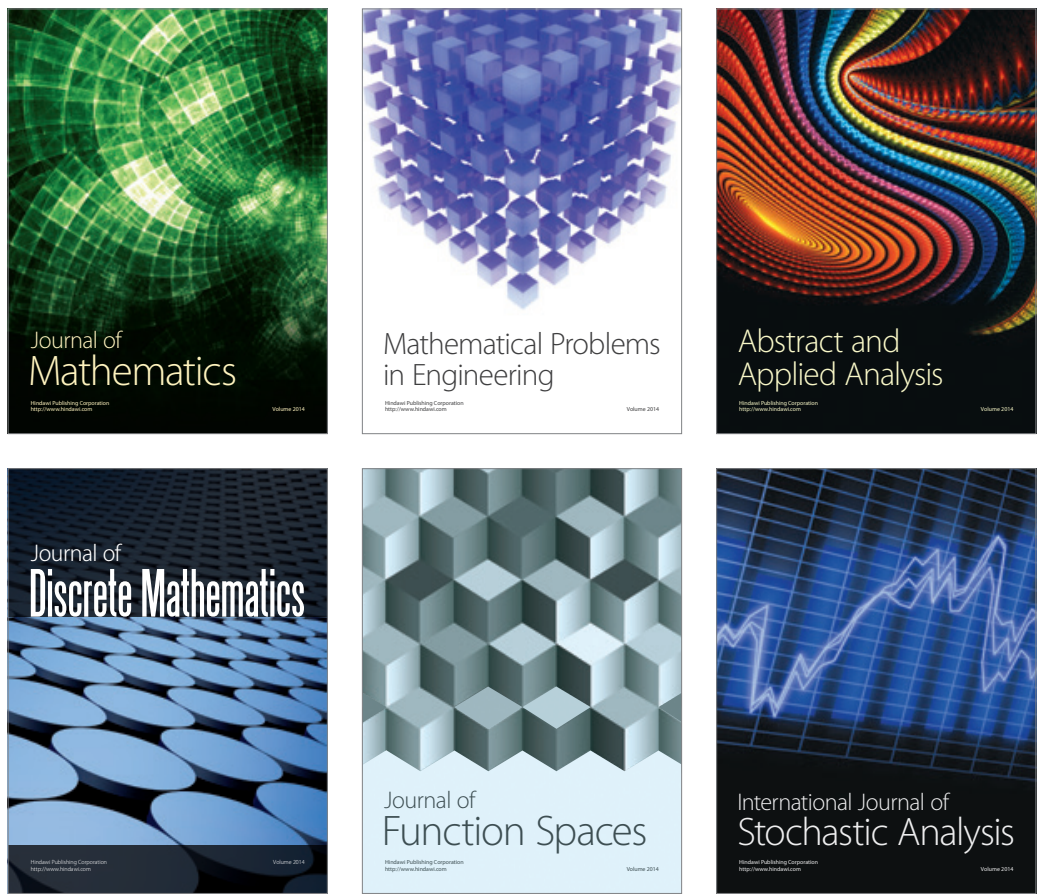

Journal of

Function Spaces

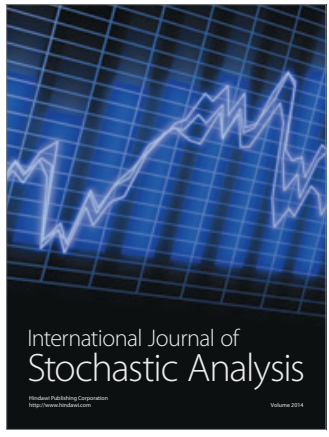

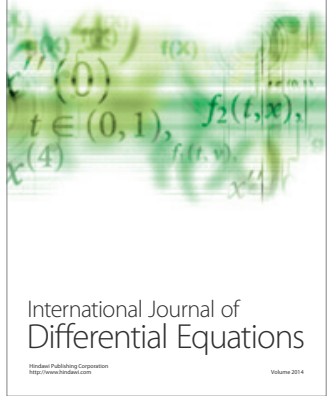
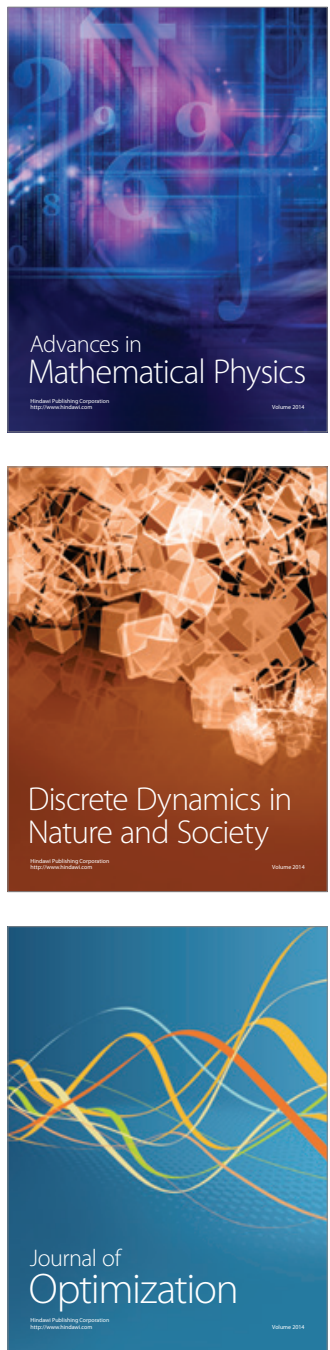